\title{
Characteristics of Elderly User Behavior on Mobile Multi-touch Devices
}

\author{
Susumu Harada, Daisuke Sato, Hironobu Takagi, and Chieko Asakawa \\ IBM Research - Tokyo \\ NBF Toyosu Canal Front Building, 5-6-52 Toyosu, Koto-ku, Tokyo 135-8511 Japan \\ \{haradas, dsato, takagih, chie\}@jp.ibm.com
}

\begin{abstract}
Smartphones and tablet devices have been rapidly proliferating, and multi-touch interaction, powerful processors and rich array of sensors make these devices an attractive service platform for older users. While there is an increasing number of work investigating the issues that elderly users experience through their interaction with mobile devices, most have focused either on evaluation of low-level interaction characteristics or on qualitative survey. Therefore, we conducted a user study with 21 elderly participants to analyze the needs and issues faced by this user group under naturalistic usage scenarios. Specifically, we interviewed each participant about their experiences, had them perform various practical tasks using our custom testing application, and analyzed the operation logs using our custom visualizations. Based on our results, we summarize the types of issues observed, present design considerations for the applications studied, and future research directions.
\end{abstract}

Keywords: Mobile, Multi-touch, Smartphones, Tablet, Aging, Elderly.

\section{Introduction}

Over the past several years, mobile multi-touch devices such as smartphones and tablets have been rapidly proliferating. While the primary growth has been among younger users, there is a growing number of elderly people who are beginning to adopt such new technology [1]. The direct manipulation interaction afforded by their multi-touch displays, their ability to run numerous applications both on the device as well as over the network, combined with powerful processors and a rich array of sensors, make these devices an attractive platform for making a wide range of services available to benefit older users.

While the multi-touch interfaces enable intuitive direct-manipulation interaction that mimic real-world metaphors, they also introduce a number of potential challenges, such as non-intuitive multi-finger gestures, unexpected sensitivity of the touch surface, and a conceptual model that differ significantly from traditional desktop computers and other preceding technologies.

Younger users may be able to quickly learn to navigate around such challenges through trial-and-error and relying on their mental models of recent technologies, but the hurdle may be bigger for older users. A study by Docampo Rama, Ridder, and 
Bouma [2] indicates that generational difference in exposure levels to various technology during one's formative years (before the age of 25) has a significant effect on the performance on and the level of understanding of new technologies. A survey with 131 respondents from three age groups $(20-49,50-64,65+)$ conducted by Leung and others [3] suggests that significantly fewer older people choose trial-and-error as a method for learning new technology compared to younger people, opting for more traditional methods such as instruction manuals despite citing difficulties using them. It is clear that the needs and characteristics of the older population are quite different from those of the younger population in the context of mobile device usage. In our investigation, we focus on the issues surrounding the usability of such devices from the perspective of elderly users.

There have been many works seeking to better capture the needs and characteristics of older users and their interaction with mobile devices. On the one hand, there have been various interview and questionnaire studies [3-7] that have identified characteristics unique to the elderly user population. While these interview and questionnaire studies provide valuable insight into elderly user's attitudes towards and perceptions of mobile devices, they do not reveal much about the interaction tendencies of and challenges faced by elderly users throughout their actual use of such devices.

On the other hand, there have also been works that sought to capture more concretely the patterns and tendencies of elderly users' interaction with mobile touch-screen devices through measurement and analysis of low-level interaction metrics such as task speed and accuracy [8-13]. Most of these works involved experiments in which the participants performed primitive actions such as tap or swipe gestures in isolation within their custom test applications. While these studies provide concrete data, the data may not be representative of what users experience in actual usage of mobile devices, since real tasks require not only primitive gesture skills but also skills to develop strategy to complete tasks by effectively selecting a series of gestures in real-time, in-situ, within the context of full application user interfaces.

In our work, we build upon the above studies by combining an observational study approach with an experimental approach that include detailed instrumentation of elderly users' interaction with mobile touch-screen devices as they perform tasks within realistic application contexts. Our aim was to uncover issues that elderly users may encounter during their typical usage of smartphones and tablets, such as errors, unexpected results, standstills, ineffective operational strategy and so on, and delve deeper into each issue by analyzing the instrumented log data.

We conducted a user study with 21 elderly participants with diverse experience levels, from first time users to active intermediate level users, to observe and analyze specific issues and challenges that they experience when using mobile touch-screen devices. We built a custom experiment application for multi-touch smartphones and tablets that mimicked the appearance and functionality of actual applications, augmenting them with instrumentation capability to capture and log all multi-touch events and application state changes. Our focus, however, was less on statistically analyzing aggregate results but more on carefully observing interaction characteristics 
such as errors, unexpected results, operational strategies and so on, of each individual participant. We also conducted interviews with each participant to solicit their comments and better understand their perceptions and past experiences with mobile devices. Based on these results, we present a number of design considerations and future research directions for creating smartphone and tablet devices and applications that are more accessible and easily adoptable by elderly users.

Our contributions are as follows:

- Quantitative analysis of elderly users' interaction behaviors within realistic application settings

- Qualitative analysis of elderly users' specific tendencies and obstacles encountered during their interaction with multi-touch smartphones and tablets

\section{Related Work}

There are many prior work that have investigated elderly users' perceptions and use of traditional mobile phones [14-17], as well as those that have examined elderly users' interactions with large touch-screen displays, but not much work has yet looked into elderly people's use of mobile touch-screen devices such as recent multi-touch smartphones and tablets.

\subsection{Elderly Users and Mobile Phones}

A number of interview and questionnaire studies have been conducted to investigate the general perceptions and practices of elderly users regarding mobile phones, and several resources provide a comprehensive overview of the literature $[1,18]$. We present some of the representative works here.

Kurniawan [4] conducted a set of expert interviews with two domain experts, focus group discussions with 14 elderly participants, and an online questionnaire that resulted in 100 responses from people over 60 years old. The study reports on various findings about elderly people's perceptions of and issues with mobile phones, such as their preference for features that aid their declining functional abilities. Leung and others [3] also conducted a large-scale online survey with 131 respondents across three age groups (20$49,50-64,65+)$ to identify unique tendencies among the older population, including their preference for traditional methods such as instruction manuals for learning new technologies over trial-and-error. Van Dyk, Renaund, and van Biljon [7] conducted an extensive interview with 147 mobile phone users between the ages of 60 and 89 to construct a prioritized checklist of features that should be considered in the design of mobile phones catered to the elderly users.

There have also been some observational studies as well as experimental studies that delve into more detail about the elderly's use of such technologies. Renaud and Biljon [6] used worth-centered design process to interview and conduct a participatory design experiment with elderly users, resulting in a prototype mobile phone design. Weilenmann [19] conducted an observational study of elderly users learning to enter text on mobile phones, highlighting specific issues they encountered. Ziefle and Bay 
[13] conducted comparative evaluation of two mobile phones of different complexity between younger and older participant groups. Jastrzembski and Charness [8] proposed and validated the parameters of a Model Human Processor for older adults.

While these studies provide valuable insight into the overall tendencies and preferences of elderly people as well as their usage characteristics within the context of mobile phones in general, they have not focused on issues that surround the more recent mobile multi-touch devices.

\subsection{Elderly Users and Touch-Screen Displays}

Touch interaction is one of the key features that differentiate recent smartphones and tablets from prior mobile devices. Leonardi and others [20] conducted experiments with a tabletop touch panel interface and found that the direct interaction metaphor was easy to understand and had a pleasing effect that attracted and motivated elderly participants in their study. Lepicard and Vigouroux [21] had a group of younger and older participants interact with their test application on a 12" Tablet PC and found that two-handed touchscreen input was difficult for elderly users. There have also been other observational studies of elderly people's use of touchscreen interfaces [22, 23], and a survey of other work is provided by Caprani, O'Connor, and Gurrin [14].

While the results from these studies offer insight into the issues that elderly users experience with touch-screen devices, there are several key differences between tabletop and desktop touchscreen devices and mobile multi-touch devices that warrant further investigation looking specifically at the latter category of devices. For one, the smaller form factor of mobile devices mean that the users are often holding the device with one hand while they touch the screen with the other hand or even the same hand. This factor, combined with the multi-touch sensing, can potentially lead to greater chances of inadvertent or accidental touches of the screen by the holding hand or some other part of their manipulating hand. Furthermore, the smaller screen estate can lead to the need for more touch manipulation to view the desired information as well as potential errors when touch targets are too close or too small. Therefore, more research is needed to investigate the more recent multi-touch smartphones and tablets.

\subsection{Elderly Users and Mobile Multi-touch Smartphones and Tablets}

While still few in number, there have been recent works that have specifically focused on mobile multi-touch smartphones and tablets as the target of their study. One part of the extensive study conducted by Leung and others [3] involved a field study observing two middle-aged and four older users learning to use smartphones. Kobayashi and others [9] observed 20 participants in their 60s and 70s using various applications on multi-touch smartphones and tablets. They also conducted controlled experiments involving execution of primitive gestures on smartphones and tablets, finding that the elderly participants' performance of dragging and pinching gestures improved after one week of practice but not tapping. Leitão and Silva [10] used a custom "game" on a multi-touch smartphone to analyze the effects of target size and spacing on tapping and swiping tasks. Nicolau and Jorge [11] evaluated typing performance and typing 
patterns of 15 participants over the age of 65 using a virtual keyboard on a multitouch smartphone and tablet.

Our goal was to build upon these works by observing and looking for specific challenges, stumbling points, and possible coping strategies employed by individual users. While our results do not provide statistical significance or sweeping generalizations, we believe that the concrete observations, both qualitative as well as quantitative, situated within the context of realistic application usage, provide valuable insight into specific issues that elderly users may encounter in their typical interaction with multi-touch smartphones and tablets.

\section{User Study}

We conducted seven half-day-long user study sessions with a total of 21 elderly participants to investigate their perceptions of smartphones and tablets as well as specific usage characteristics as they performed tasks on the devices. Our goals were:

1) to better understand elderly users' perception of smartphones and tablets,

2) to identify "realistic issues" encountered by elderly users as they interacted with real and "realistic" apps,

3) to quantitatively analyze the issues identified in 2) to see if we could systematically identify and potentially circumvent such issues, and

4) to uncover design considerations that could inform design of more senior-friendly smartphones and tablets.

\subsection{Participants}

We recruited participants for our study through email announcements distributed to retiree mailing list for a large corporation, as well as to local computer classes for seniors. The condition for participation was specified as being 60 years of age or older with some interest in smartphones and tablet devices.

A total of 21 participants ranging in age from 63 to 79 (12 females and 9 males) took part in our study. Fifteen of the participants were members of the local computer class, and six were from the retiree group. The participants were divided into seven groups, each consisting of two to four members, so that we could conduct a focus-group-style discussion. Each group was invited to our lab for a half-day session, and each participant was compensated for their time with a gift valued at approximately $\$ 25$. All of the participants owned a mobile phone. Eight of them owned multi-touch smartphones while 14 owned a tablet, and six owned both devices.

\subsection{Procedure}

Each of the half-day user study sessions was structured into two parts: 1) open discussion about each participant's experiences with and perceptions of smartphones and 
tablets, and 2) execution of controlled tasks on smartphones and tablets. The sessions were audio recorded for later analysis, and with participants' permission, video was also recorded to capture the user's hands operating the devices during various tasks. During each session, two experimenters were present, one to primarily engage with the participants, and another to take notes and to tend to the video cameras.

Open Discussion. At the start of the user study session, we first had all participants individually fill out a questionnaire soliciting demographic information as well as specifics about their experiences with smartphones and tablets. In particular, we prepared different questionnaires for those who did or did not own a smartphone or a tablet. To those who did not own the devices, we asked whether they had considered owning one, and if so, what factors had interested them and what factors had prevented them from actually owning one. To those who owned the devices, we asked for their reasons for purchasing them and what they most use them for. After the questionnaires were filled out, about 30 minutes was spent on open group discussion about each participant's experiences and perceptions about smartphones and tablets as well as their current mobile phones. The discussion was led by the experimenter based on a prepared set of guiding questions, but the participants were free to expound upon or add any stories about their experiences. The group discussion format was adopted so that the participants were able to engage in a more casual conversation, feeding off of each other's responses and stories.

Controlled Task Experiment. In the controlled task experiment session, we had the participants perform various tasks on both the smartphone and tablet using the data collection application we built to gather logs of quantitative usage data. The purpose of this session was to attempt to gain an objective view of any tendencies or unique characteristics in the interaction styles of each participant in using various touchoperated apps. The primary objective of this investigation was not to obtain statistically significant aggregate data, but rather to observe and analyze the usage patterns of individual participants so that we may gain specific insight into concrete manifestations of errors, operation difficulties, and other issues. Ideally we would have liked to evaluate the usage of real applications under actual usage scenarios, but we resorted to an in-lab study to more closely observe the interaction issues as they happened.

Each participant performed in an order of Address Book, Phone, and Map tasks on both smartphone and tablet. The order of devices was counterbalanced. All participants performed the same set of tasks on both devices. For the Phone task, they input three 11-digit phone numbers printed on a sheet paper. For the Address Book task, the participant was presented with a same list of 160 names but with different initial starting position in the list, and were prompted to search for and tap on a particular name. A total of 10 pre-chosen names were presented one by one and were shown at the top of the application. For the Map task, the participants were asked to find three locations (Hokkaido, Okinawa, and England) on a map, zooming in to fill up the screen with the target island as the completion condition for each trial. The map was reset to show the Tokyo Bay area at the beginning of each trial. While not all apps required multi-touch gestures, we wanted to see if the multi-touch-enabled screen would lead to various errors caused by 
unintentional touches during the tasks, a common symptom observed especially among novice multi-touch device users.

\subsection{Apparatus for the Controlled Task Experiment}

For the controlled task experiment, we used Apple's iPod Touch (fourth generation) and iPad (third generation). Both devices were running iOS version 5 . We built a custom test application that included three application interfaces that closely mimicked the appearance and behavior actual applications found on standard iOS devices (Phone, Address Book, and Map). The test application logged all multi-touch events and application events for later analysis.

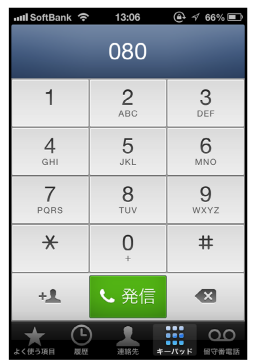

Phone

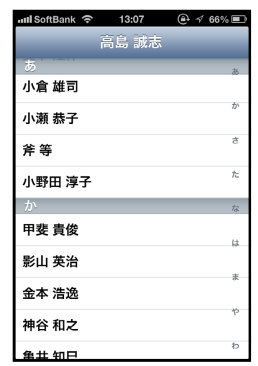

Address Book

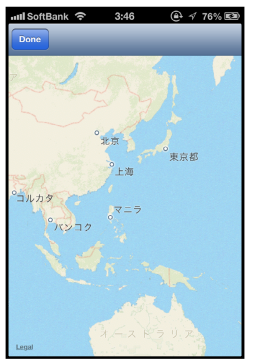

Map

Fig. 1. Screenshots of the three application interfaces in our test application

Phone Application Interface. The phone application interface consisted of the standard telephone keypad, and an entry display at the top showing the numbers entered. The actual visual of the user interface was directly copied from the original application. The app was set up such that after the participant entered the correct phone number for a particular trial and tapped the dial button, the screen returned to the main screen. If the user tapped the dial button but the phone number entered was incorrect, a dialog was displayed to prompt the user to re-enter the correct number.

Address Book Application Interface. The address book application interface consisted of a vertical list of contact names sorted and grouped alphabetically, with an index bar on the right side of the screen that support jumping to the section of the list corresponding to the tapped index letter. During each task trial, the participant was first presented with a dialog displaying the target name to find in the contact list. After dismissing the dialog, the target name remained visible at the top of the screen as the user scrolled through the list.

Map Application Interface. The map application interface simply presented the standard iOS map control, with the ability to use two-finger pinch-in and pinch-out gestures to zoom out and in, respectively, as well as to pan the map using either one or two fingers. Double tapping with a single finger to zoom in by a fixed amount and single tapping with two fingers to zoom out by a fixed amount were also possible. 


\section{Controlled Experiment Results}

\subsection{Phone Application Task Results}

Position of Touches. Participants completed each trial on average in 13.2 seconds on the smartphone and in 11.9 seconds on the tablet. For each of the $63(3 \times 21)$ trials on smartphone and tablet, the numbers of incorrect touches were 32 and 15 in total, respectively. Figure 2 shows the plots of correct touches and incorrect touches made by all participants on both devices. With the tablet, five participants made incorrect taps with their palm, which can be seen at the bottom-right of the plot for the tablet. With the smartphone, three participants made an incorrect "call" while intending to tap " 0 ". Most of the other incorrect taps were due to misreading the phone numbers.

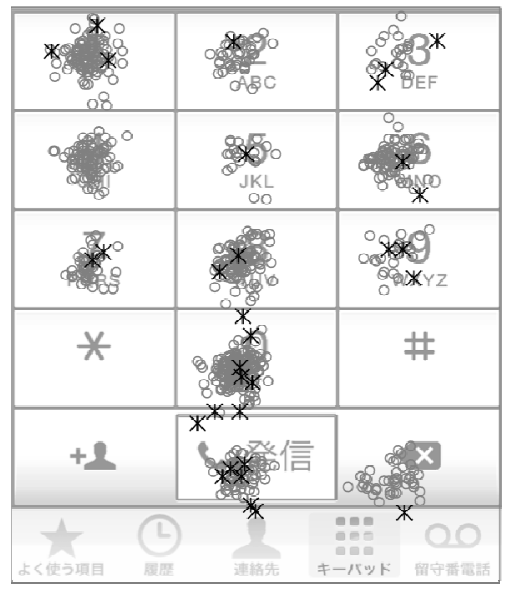

Smartphone

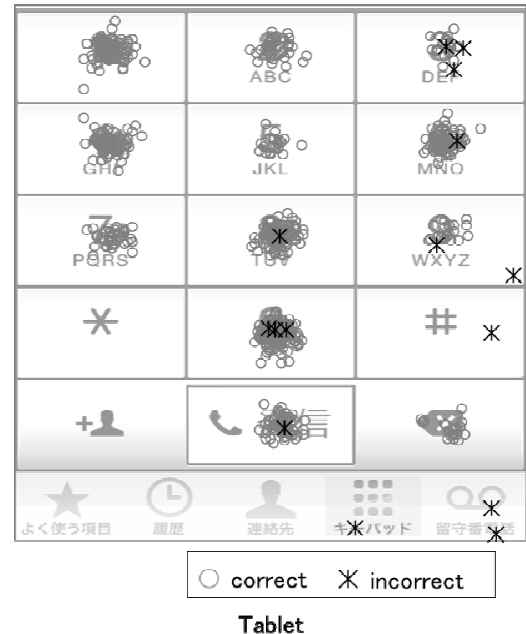

Tablet

Fig. 2. Correct and incorrect touches in the Phone task on smartphone and tablet

We observed P13 input one extra digit with an unexpected repeat of the " 8 " button. By checking the recorded video for the trial, she did only tap once on the " 8 " button but the system accidentally recognized two distinct taps within 0.13 seconds. Also many unregistered taps were observed, in which participants physically tapped on the screen but the system failed to recognize them.

Not Confirming before Moving on. There was an instance when P13 tapped the "call" button with one too many digit entered, and the dialog prompted her to correct the entry. She then tried to re-input the number from the beginning but only tapped the delete button once, not checking the number display at the top, resulting in an even longer sequence of numbers. P04 and P15 also got the dialog prompt during the task by tapping "call" button with too few digits of input. After they dismissed the dialog, they also tried re-entering the digits from the beginning without deleting or checking the display at the top of the screen. 


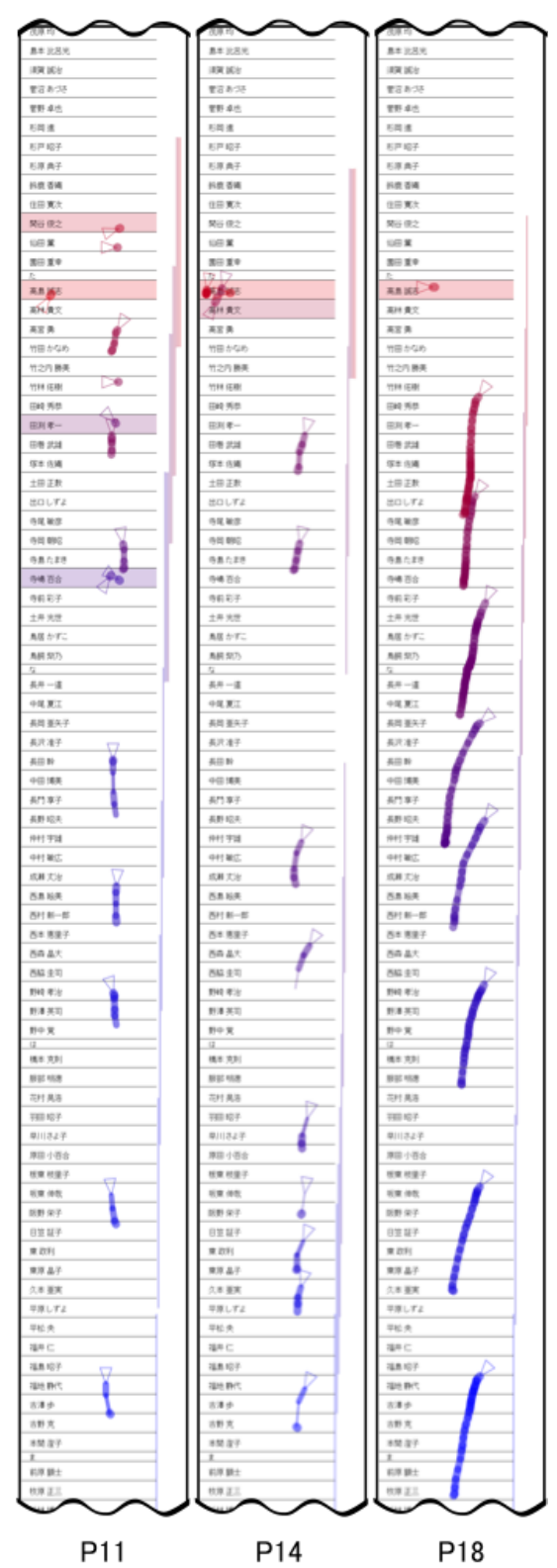

Fig. 3. Trajectories of all gestures within representative trials for the scroll task on the smartphone across three participants. (Nudge-flicking: P11 and P14, Strokeflicking: P18)

\subsection{Address Book Application Task Results}

Participants completed each trial on average in 9.7 seconds on the smartphone and in 8.9 seconds on the tablet. P09 and P11 made 15 and 12 miss-taps, respectively, significantly more than the maximum of four miss-taps made by the other participants. Most of the mistakes by P09 on the tablet were missselection of list item while intending to tap on the index bar. All miss-taps by P11 were on the smartphone, and seemed to be due to her hand tremor.

Difficulties with the Index Bar. P4, P5, P9, P18 and P23 experienced a number of instanced in which they unintentionally manipulated the index bar. In particular, the participants were especially confused when they were attempting to drag the list vertically but instead accidentally started their gesture on the index bar. As they moved their finger, the list jumped discretely across the index groups instead of smoothly following the participant's finger movement. P4, P5 and P9 experienced these issues even though they were aware of the index bar function previously. P4 also exhibited confusion when she tapped the index bar my mistake when attempting to tap the target row.

Similar to the case with the Phone application interface, we also observed a number of unintentional touches primarily by the participants' palms. Due to the index bar being on the right side of the screen and all participants being right handed, this often led to the index bar being accidentally tapped, resulting in the list jumping to a grouping unexpectedly. As a result, P23 commented, "the screen just 'flew away'... I just touched it lightly just like this, and then it just flew away somewhere..." 


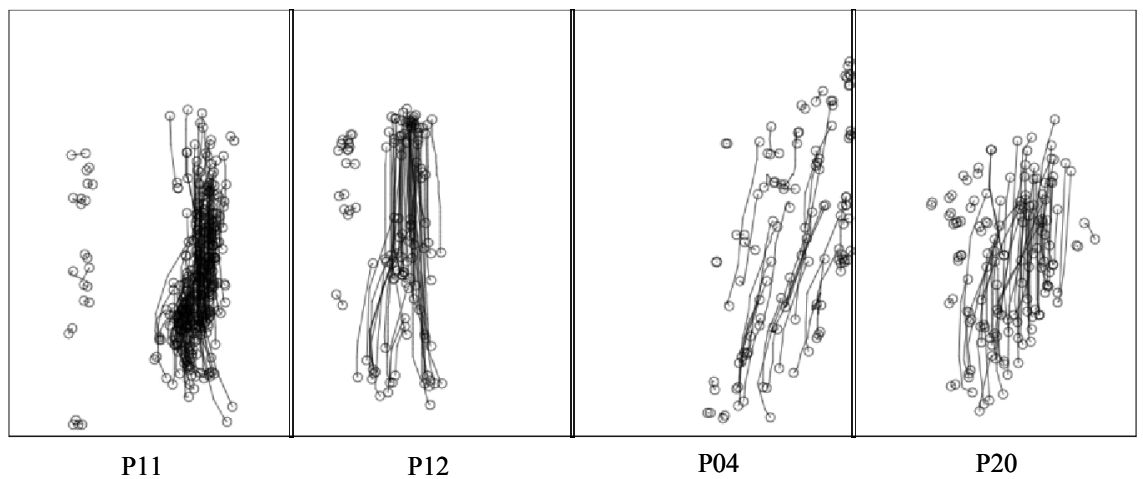

Fig. 4. Trajectories of all gestures for the Scroll task on the smartphone

Nudge-Flicking Instead of Stroke-Flicking. There were distinct differences among the participants in their finger movements for scrolling the list up or down. It is typical for experienced users to use short, quick "flick" gestures to "throw" the list towards a given direction to take advantage of the inertial scrolling to scroll over a long distance, and to use controlled "dragging" gesture to keep the list pinned below the firmly touching finger to move the list by a small amount. Figure 3 shows a visualization of users' gestures during a particular trial of the Address Book task. Each stroke represents the user's gesture on the screen. Triangles at the beginning of each stroke represent the stroke direction, and rows that were tapped, including both correct and incorrect selections, are shown filled. While most users where able to utilize inertial scrolling, P11 and P14 in particular used extremely short strokes for flicking, only moving the tip of the finger to perform the gesture, in contrast to participants such as P18 who exhibited a much longer flicking stroke, moving the entire hand or even the arm to perform the gesture.

Figure 4 shows the trajectories of all gestures across all trials on the smartphone by P11, P12, P04, and P20. P11 and P12 taped on the left side of the smartphone when selecting a name on the list. Each list item has a full screen width, and all participants except P4 and P20 tended to tap on the side of the screen with the names displayed.

\subsection{Map Application Task Results}

Participants completed each trial on average in 23.8 seconds with the smartphone and in 23.7 seconds with the tablet. Figure 5 shows a visualization of users' operations as they navigated from Tokyo to England on the map (P6 on the tablet and P15 on the smartphone). Each rectangle in the visualizations shows what the device showed when they performed the corresponding gestures and the solid lines within the rectangles represent the gesture trajectories. They used zoom-out gestures but the zoom-out level was not enough to explorer the world map, and they tried to find the goal (England) by executing numerous swiping gestures. 


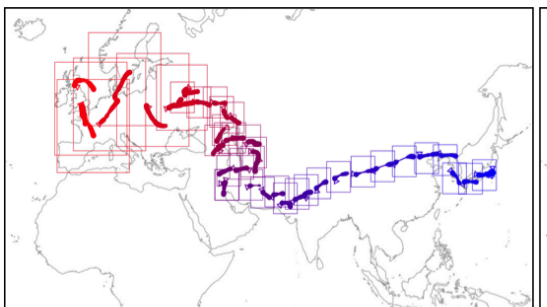

P06, tablet

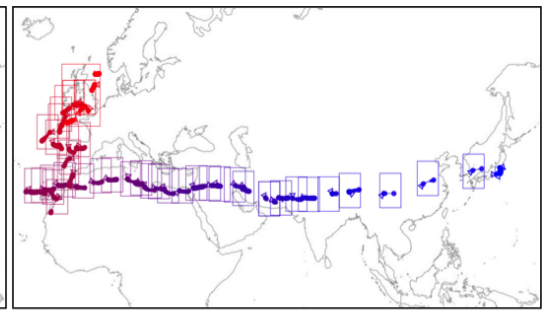

P15, smartphone

Fig. 5. Operation sequence for a particular Map task trial by P06 and P15

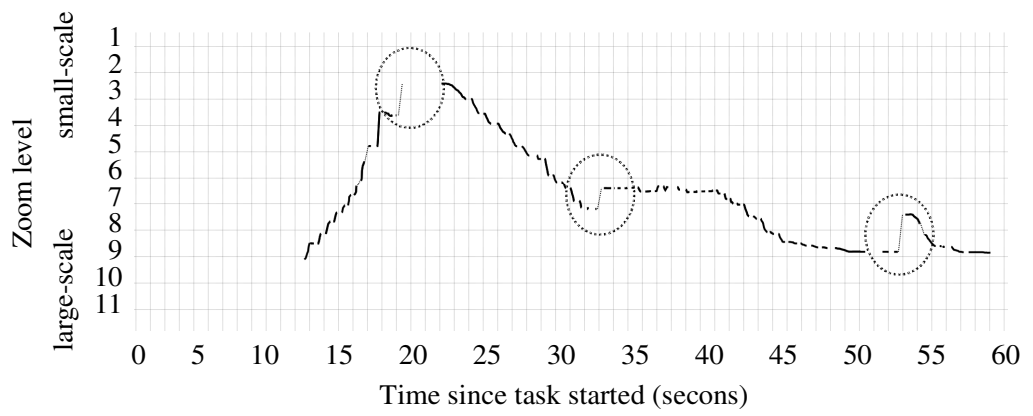

Fig. 6. Zoom level sequence for a particular Map task trial by P19

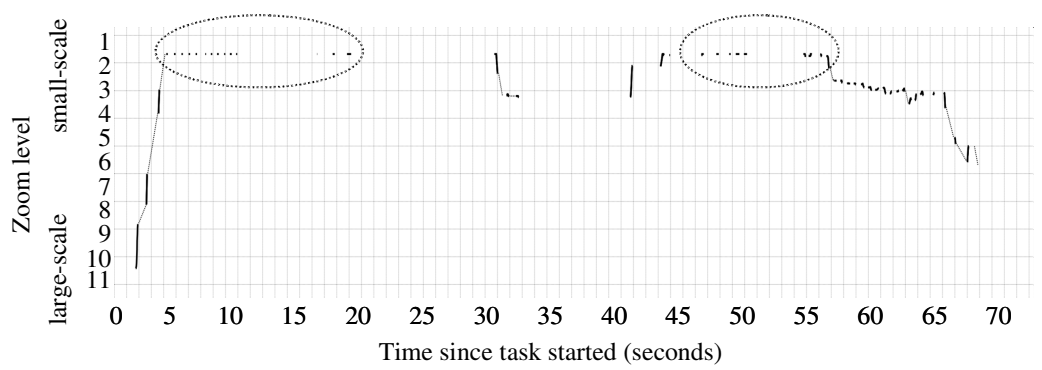

Fig. 7. Zoom level sequence for a particular Map task trial by P17

Figure 6 and Figure 7 visualizes the zoom level sequence of the task by P19 and $\mathrm{P} 17$, respectively. Vertical axis shows the zoom level from world (small-scale) to town (large-scale) and horizontal axis shows the time since the task started in seconds. Thick lines indicate zoom level change while touching and thin lines indicate zoom level change without touching (inertial, double tap, or single tap with two fingers). P19 used two fingers for both zooming and panning and each gesture was very short, causing unexpected zoom-outs during operations. Three dashed circles in 
Figure 6 indicate where the map recognized single tap with two fingers to zoom out for a certain step. This type of miss operations was also found in P13, P14, P15, P17, P20, P21 and P23. Two dashed circles in Figure 7 show that P17 attempted many pinchin (zoom out) gestures at the minimum scale. He was aware that something was not going as expected (i.e., the map was not zooming out any further than its current level), but he did not stop executing the zoom-out gesture.
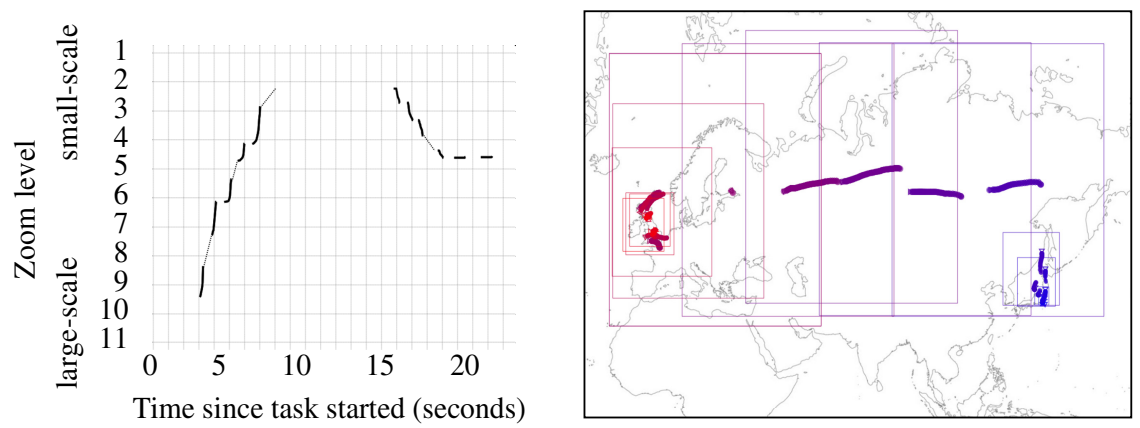

Fig. 8. Operation sequence for a particular Map task trial by P23

Figure 8 shows a good example of operation by P23 from Tokyo to England. He could zoom out smoothly and easily found the target after a few swipes and then zoomed into the target. By comparing such participants' log traces, it becomes clear that there are distinct differences in the characteristics of the performed gestures as well as their strategy in performing the task of locating a target on a map.

The smaller and incremental pan and zoom gestures were observed more among those who had less experience with smartphones and tablets (i.e., those who did not own the corresponding devices), so we looked into the differences in the ratio of zoom operations of varying magnitudes, grouped by device experience (Figure 9a). A similar analysis was performed for panning movement distance as well (Figure 9b). Figure 9a shows that people who do not own smart devices tend to use smaller scale factor of zoom-in and zoom-out. Figure $9 \mathrm{~b}$ shows that people who do not own smart devices tend to use shorter panning, most of the panning were less than 300 pixels, while people having smart devices use various length of panning.

Comparing to the other two apps, many difficulties of operations were observed with map application. P6 and P19 use more than two fingers for zoom-in and zoom-out. Unfortunately, since the tablet has a function to close application by four fingers pinch-in gesture, the experiment app was terminated sometimes by the four fingers gesture when they wanted to zoom-out on the map. Two hands were used to perform for pinch-in/out gestures by P17 and P20 on smartphone and tablet respectively. P17 had difficulty zooming with two fingers on smartphone. Many participants performed a single tap gesture on the map instead of a double tap gesture when they saw their target location at a low zoom level, seemingly in an attempt to zoom in to the target location. 


\section{$5 \quad$ Interview Results and Observations}

Here we report on other observations across the experimental tasks and through our conversations with the participants.

\subsection{Unexpected Touch Screen Responses}

There were a number of instances when a participant either intended to touch the screen but the system did not register (unregistered touches), or did not intend to touch the screen but the system registered a touch event (unintentional touches). Such unexpected responses seemed to be a major cause for frustration and confusion expressed by the participants.

Regarding unregistered touches, the primary cause seemed to be that the participants' fingers were too dry for the touch-sensitive display to detect. P4, P5, and P6 mentioned frequently having problems during their daily life with dry finger resulting in ATMs and other touch screens not responding. P14, P18, and P20 all commented during their experimental tasks that their fingers felt dry and that the device seemed not to be responding to their touches.
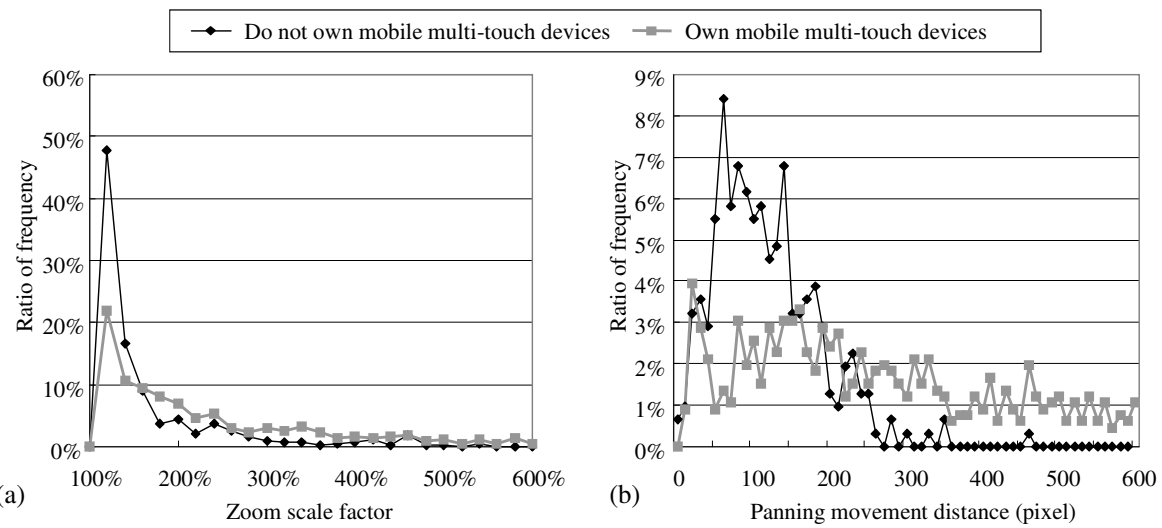

Fig. 9. (a) Comparison of frequency of zoom scale factor in the Map task across users who do and do not own mobile multi-touch devices. (b) Comparison of ratio of panning gesture lengths in the Map task across users who do and do not own mobile multi-touch devices.

Unintentional touches were also observed across many participants. There were several patterns of unintentional touches. First was when the participant's non-controlling hand that was holding or resting near the device accidentally touched the screen surface. Another was when the participant was hovering above the touch screen surface contemplating what to tap, and got too close to the surface of the display and triggered a touch event. Finally, there were cases when the user was performing some gesture such as a single tap or a swipe, during which some other part of their hand also accidentally contacted the display surface, resulting in multi-touch events being registered. 
P10, P13, and P14 mentioned that they often experience situations in which unintentional touches are triggered by the sleeve of their clothing making contact with the touch screen at ATMs or when using their tablet. While we do not know for certain whether it is actually the sleeve of their clothing that was making contact with the touch screen or a part of their hand, but it is clear that unintentional touches are quite a common phenomenon that is noticed by these elderly users. P6, P11, P12 and P22 all mentioned that they experienced difficulty with such unintentional touches when they first purchased their touch screen devices but that they had now gotten used to it.

\subsection{Not Seeing the State of the Entire Screen}

A number of participants tried to start scrolling before dismissing the popup dialog in the address book task. In the phone app, when using the backspace button, some users seemed to assume that it deleted not just the most recent digit but all digits entered so far. When such a user miss-entered a digit in the middle of a sequence, they tapped the delete button once but then proceeded to reenter the entire number sequence from the first digit, and not being aware of their mistake as they did not seem to be checking the number display at the top.

During the interview session, we observed some users entering text into a search textbox. During this process, these users were focused on the on-screen keyboard keys, and were not looking at the actual text being entered in the text box, which was at the upper left corner of the screen. Unlike physical keyboard where touch-typing is possible due to the tactile feedback from the physical keys, touch-screen keyboard inherently requires user to direct visual attention to each key the user is about to tap. For elderly, seems especially challenging to shift attention back and forth from keys to the textbox where text is being entered. As a result, they type away until they finish their phrase, and only then go to check what they typed, which may include surprising results due to mistyped characters and auto-suggestions.

\section{3 “Unfriendly" Interface}

During the interview session, a number of participants tried entering text into a memo application using the on-screen keyboard. There were several keyboard layouts available, and to switch among them, a particular button had to be pressed. However, if the user stays in contact on that button for slightly longer than a quick tap, to bring up a popup menu with a list of available keyboards is shown. The popup, however, automatically fades away after a few seconds. P3, P4 and P5 had difficulty with this, as they would tap the button once, and before they could finish reading the list of options and tap on a selection, the popup faded away.

Tap-and-hold menus are tricky, especially because by holding the finger down, it may be visually covering the available options. Tap should show the menu and keep it shown, but the issue is, how to dismiss the menu without making a selection. Tapping "outside" the menu should be used, but sometimes, since the background behind the popup menu is still highly visible, one may be reluctant to tap on the "background" for 
fear of activating something else. A clearer indication of which part of the interface is actionable at any time may be needed.

\subsection{The "Aha" Moments When Being Shown a New Technique}

After the controlled task experiments were completed, the experimenters debriefed the participants by describing and demonstrating some techniques for performing some of the interactions more accurately and precisely. There were a number of instances when users expressed particular surprise and joy at learning about such new method of interaction that was more effective than what they had been used to. For instance, P4 and P5 were excited to be shown that the device can also respond with the side of the finger instead of just the tip. P10, P11, and P12 also expressed excitement at learning how to perform the pinch-in gesture on the map by not flicking the two fingers together but by assuming that the map is like a rubber sheet in which the points under the fingers remain fixed as the fingers are moved.

As elderly people may be less reluctant to experiment [3], it may be good to show various alternative interaction approaches, preferably gradually and repeatedly, to accommodate for their slower learning pace.

\section{Discussion}

This section describes a number of design considerations for enhancing the three applications we used in our study, based on the above results, to address some of the issues encountered by our elderly participants.

\subsection{Phone Application Interface}

As described in Section 4.1, the main issues experienced by the elderly participants in the phone application interface were accidental taps of buttons adjacent to the target buttons, especially problematic between the "zero" and the "call" buttons, and also not being aware of the top part of the screen showing the phone number. The issues of unexpected touch screen responses are one of major issues across applications (Section 5.1).

One way to mitigate these issues may be to provide better feedback through both audio and visual. The phone application currently provides dial-tone feedback whenever a key is pressed, but this could be enhanced by having the tapped digit be spoken out. Work by Sato and others [24] have shown that such spoken feedback during online web form entry helped elderly users feel more confident and reassured. Such feedback mechanism will help various situations such as misrecognition caused by "dry" finger or cloths (Section 5.1) In addition to sound feedback, visual feedbacks may be effective. The phone application also highlights the pressed button. In addition to this visual feedback, showing a fading "fingerprint" on the display to show the actual touch position may be helpful for the users. This could be combined with auditory icon and/or color feedback to indicate number of touches detected. 
Furthermore, the application could determine whether the input number is a valid phone number or not to change behavior of the "call" button dynamically. For example, if the phone number is not valid, the system can show a confirmation dialog to the user. More implicit way to handle the situation may be for the system to ignore the press of the "call" button once but letting the second press invoke the actual call.

\subsection{Address Book Interface}

Although using the index bar to navigate to an entry in a list can be effective (Section 4.2), a number of our participants experienced difficulties in manipulating the index bar. Some participants unintentionally tapped on a row entry instead of on the index bar, some participants operated index bar before dismissing the popup dialog (Section 5.2) and some participants touched the index bar at the beginning of their swipe gesture when scroll the list (Section 4.2). There may be several reasons for these mistakes, including the index bar being too narrow, the touchable area of the index bar not being visible (it temporarily becomes visible while the user is touching the index bar), and the index bar being laid out on the right edge of the screen. One possible solution for the current index bar design is to place the bar on the left edge of the screen for right-handed users and making the labels bolder and more visible. If this design is not acceptable from an aesthetic point of view, the index bar may be better off being removed altogether. As the scroll list component handles both tap gestures as well as swipe gestures, there is a greater possibility that the user's gesture may be misinterpreted compared to the phone application that only handles tap gestures.

\subsection{Map Interface}

Under the map interface, there are more gestures available than with the address book interface, including pinch to zoom, double tap, two-finger single-tap, etc. While our map interface did not implement them, there are other map applications that support even more gestures such as tap and hold for dropping pins on the map and using two fingers to rotate the map, making maps one of the more comprehensive apps in terms of the types of gestures supported. Furthermore, the notion of an "incorrect" operation on map interface is not as clear as with the phone or the address book interfaces.

As a possibility for enhancing the map application interface for elderly users, it may be desirable to support the ability to disable or enable various gestures to match the skill level of the user. Furthermore, a training mode that teaches the user about each available gesture and assesses users' skill of gestures by analyzing their panning and zooming behaviors (Figure 9) allows the user to practice them and to be aware of the correct concept of map operation (Section 5.4). It would not only benefit the map application interface but other applications in general as well.

We also observed a participant repeatedly attempt to pinch out beyond the maximum supported zoom level (Section 4.3), but such a behavior of repeatedly attempting ineffective gestures was not seen in the address book interface. The reason could be that in the address book interface, when the end of the list is reached, any attempt to scroll further results in a bouncing animation where the list bounces back to its limit position. 
By adopting a similar animation feedback for the map's zoom function, it could more clearly communicate to the user that the attempted action is ineffective.

\section{Conclusion}

We investigated the issues and challenges that elderly people may encounter when using multi-touch smartphones and tablets by conducting a user study with 21 elderly participants. The user study sessions comprised of open discussion about each participant's experiences with and perceptions of smartphones and tablets, and an experiment involving controlled tasks on smartphones and tablets to gather interaction characteristics. By creating our logging application that captured detailed interaction logs while mimicking the appearance and functionality of actual application interfaces, we were able to observe realistic issues that the participants encountered, while also being able to visualize and analyze the details of their interaction styles and the difficulties they encountered.

Our observations revealed a number of specific issues and challenges encountered by our participants. For instance, a number of them experienced unintentional taps due to parts of their hand accidentally making contact with the touch screen, as well as unregistered taps due most likely to their fingers being dry. Our log visualizations also revealed specific interaction behaviors, such as differences in panning and zooming strategies on the map application, with some users not utilizing zooming much at all and simply panning long distances to find the desired target.

Based on these and other observations from our study, we provided some design considerations for enhancing the three applications as well as suggested methods to train and guide the user towards gaining a better understanding of the issues they may encounter and how to deal with them.

Our exploration is preliminary, but the approach we adopted of gathering detailed logs while users performed tasks on realistic interfaces can yield many interesting and insightful findings. Since "elderly users" is a very broad sweeping category of users, no one study can comprehensively cover a representative collection of users from the category. Therefore, there is a need for a greater number of studies to be conducted looking at specific issues encountered by a wide variety of users. Further work also needs to be done to explore a wider range of applications and interaction methods, to explore other ways of effectively visualizing the interaction logs, and to implement and validate the proposed enhancements.

\section{References}

1. Plaza, I., Martín, L., Martin, S., Medrano, C.: Mobile applications in an aging society: Status and trends. Journal of Systems and Software 84, 1977-1988 (2011)

2. Docampo Rama, M., Ridder, H.D., Bouma, H.: Technology generation and age in using layered user interfaces. Gerontechnology 1 (2001)

3. Leung, R., Tang, C., Haddad, S., Mcgrenere, J., Graf, P., Ingriany, V.: How older adults learn to use mobile devices: Survey and field investigations. ACM Trans. Access. Comput. 4, 11:1-11:33 (2012) 
4. Kurniawan, S.: Older people and mobile phones: A multi-method investigation. Int. J. Hum.-Comput. Stud. 66, 889-901 (2008)

5. Lee, Y.S.: Older adults' user experiences with mobile phones: Identification of user clusters and user requirements (2007), http://scholar.lib.vt.edu/theses/ available/etd-09172007-135013/

6. Renaud, K., Van Biljon, J.: Worth-centred mobile phone design for older users. Univers. Access Inf. Soc. 9, 387-403 (2010)

7. Van Dyk, T., Renaud, K., Van Biljon, J.: Moses - method for selecting senior mobile phones: Supporting design \& choice for the elderly. In: Proceedings of the South African Institute for Computer Scientists and Information Technologists Conference, pp. 277-285. ACM, New York (2012)

8. Jastrzembski, T.S., Charness, N.: The model human processor and the older adult: Parameter estimation and validation within a mobile phone task. J. Exp. Psychol. Appl. 13, 224-248 (2007)

9. Kobayashi, M., Hiyama, A., Miura, T., Asakawa, C., Hirose, M., Ifukube, T.: Elderly user evaluation of mobile touchscreen interactions. In: Campos, P., Graham, N., Jorge, J., Nunes, N., Palanque, P., Winckler, M. (eds.) INTERACT 2011, Part I. LNCS, vol. 6946, pp. 83-99. Springer, Heidelberg (2011)

10. Leitão, R., Silva, P.A.: Target and spacing sizes for smartphone user interfaces for older adults. hillside.net. (2012)

11. Nicolau, H., Jorge, J.: Elderly text-entry performance on touchscreens. In: Proceedings of the 14th International ACM SIGACCESS Conference on Computers and Accessibility, pp. 127-134. ACM, New York (2012)

12. Wacharamanotham, C., Hurtmanns, J., Mertens, A., Kronenbuerger, M., Schlick, C., Borchers, J.: Evaluating swabbing: A touchscreen input method for elderly users with tremor. In: Proceedings of the SIGCHI Conference on Human Factors in Computing Systems, pp. 623-626. ACM, New York (2011)

13. Ziefle, M., Bay, S.: How older adults meet complexity: Aging effects on the usability of different mobile phones. Behaviour \& Information Technology 24, 375-389 (2005)

14. Caprani, N., O'Connor, N.E., Gurri, C.: Touch screens for the older user. In: Auat Cheein, F. (ed.) Assistive Technologies. InTech (2012)

15. Czaja, S.J., Charness, N., Fisk, A.D., Hertzog, C., Nair, S.N., Rogers, W.A., Sharit, J.: Factors predicting the use of technology: findings from the Center for Research and Education on Aging and Technology Enhancement (CREATE). Psychol. Aging. 21, 333-352 (2006)

16. Pattison, M., Stedmon, A.: Inclusive design and human factors: Designing mobile phones for older users. PsychNology Journal 4, 267-284 (2006)

17. Morris, A., Goodman, J., Brading, H.: Internet use and non-use: Views of older users. Univers. Access Inf. Soc. 6, 43-57 (2007)

18. Zhou, J., Rau, P.-L.P., Salvendy, G.: Use and design of handheld computers for older adults: A review and appraisal. International Journal of Human-Computer Interaction 28, 799-826 (2012)

19. Weilenmann, A.: Learning to text: An interaction analytic study of how seniors learn to enter text on mobile phones. In: Proceedings of the SIGCHI Conference on Human Factors in Computing Systems, pp. 1135-1144. ACM, New York (2010)

20. Leonardi, C., Albertini, A., Pianesi, F., Zancanaro, M.: An exploratory study of a touchbased gestural interface for elderly. In: Proceedings of the 6th Nordic Conference on $\mathrm{Hu}-$ man-Computer Interaction: Extending Boundaries, pp. 845-850. ACM, New York (2010) 
21. Lepicard, G., Vigouroux, N.: Touch screen user interfaces for older subjects: Effect of the targets number and the two hands use. In: Miesenberger, K., Klaus, J., Zagler, W., Karshmer, A. (eds.) ICCHP 2010, Part II. LNCS, vol. 6180, pp. 592-599. Springer, Heidelberg (2010)

22. Caprani, N., Doherty, A.R., Lee, H., Smeaton, A.F., O'Connor, N.E., Gurrin, C.: Designing a touch-screen sensecam browser to support an aging population. In: CHI 2010 Extended Abstracts on Human Factors in Computing Systems, pp. 4291-4296. ACM, New York (2010)

23. Tsai, T.-H., Chang, H.-T., Wong, A.M.-K., Wu, T.-F.: Connecting communities: Designing a social media platform for older adults living in a senior village. In: Stephanidis, C. (ed.) Universal Access in HCI, Part II, HCII 2011. LNCS, vol. 6766, pp. 224-233. Springer, Heidelberg (2011)

24. Sato, D., Kobayashi, M., Takagi, H., Asakawa, C., Tanaka, J.: How voice augmentation supports elderly web users. In: The Proceedings of the 13th International ACM SIGACCESS Conference on Computers and Accessibility, pp. 155-162. ACM, New York (2011) 